Recherches sur les Vitaceae du Shaba (Zaïre) III. Cyphostemma (Planch.) Alston nouveaux pour la flore du Zaïre

Author(s): F. Malaisse and M. Matamba

Source: Bulletin du Jardin botanique National de Belgique / Bulletin van de Nationale Plantentuin van België, Dec. 31, 1990, Vol. 60, No. 394 (Dec. 31, 1990), pp. 307-316

Published by: Botanic Garden Meise

Stable URL: http://www.jstor.com/stable/3668222

JSTOR is a not-for-profit service that helps scholars, researchers, and students discover, use, and build upon a wide range of content in a trusted digital archive. We use information technology and tools to increase productivity and facilitate new forms of scholarship. For more information about JSTOR, please contact support@jstor.org.

Your use of the JSTOR archive indicates your acceptance of the Terms \& Conditions of Use, available at https://about.jstor.org/terms 


\title{
Recherches sur les Vitaceae du Shaba (Zaïre) III. Cyphostemma (Planch.) Alston nouveaux pour la flore du Zaïre
}

\author{
par
}

\author{
F. Malaisse \& Matamba M. (*)
}

Abstract. - Three Cyphostemma with Zambezian distribution are reported from Zaire for the first time; furthermore, a new noteworthy species with simple leaves is described.

$\mathrm{Au}$ cours de nos recherches floristiques et écologiques au Shaba nous avons récolté plusieurs espèces de Cyphostemma (Planch.) Alston non encore signalées du Zaïre. Leur description et leur distribution sont données ci-dessous. Nous décrivons également une espèce nouvelle dont l'étude nous fut confiée.

1. Cyphostemma septemfoliolatum Wild \& Drummond, Kirkia 2: 138 (1961), Fl. Zamb. 2: 472 (1966). - Fig. 1.

Herbe vivace, dressée, de 0,5-1 $\mathrm{m}$ de haut, à racine principale pivotante pouvant atteindre $2 \mathrm{~cm}$ de diam. et $\pm 15 \mathrm{~cm}$ de longueur et à quelques racines latérales à obliques portant des sphères tubéreuses pouvant atteindre $4 \mathrm{~cm}$ de diam.; tige vertbrun, simple, pubescente dans le jeune âge et devenant progressivement glabrescente, à stries longitudinales superficielles et à section circulaire, à vrilles généralement absentes. Feuilles 5-9, discolores, composées-digitées, (1-3)-5-7 foliolées; stipules triangulaires à lancéolées, de 2-7 $\mathrm{mm}$ de large à la base et $8-20 \mathrm{~mm}$ de

(*) F. Malaisse, Laboratoire d'Ecologie, Phytogéographie et Phytosociologie, Faculté des Sciences agronomiques, B-5030 Gembloux (Belgique) et Jardin botanique national de Belgique, Domaine de Bouchout, B-1860 Meise (Belgique); Matamba M., Laboratoire de Botanique et d'Ecologie, Université de Lubumbashi, B.P. 1825, Lubumbashi (Zaïre). - Manuscrit déposé le 27 août 1990. 
long, glabrescentes; pétiole pubescent pouvant atteindre $4(6) \mathrm{cm}$ de long; folioles linéaires-elliptiques à très étroitement elliptiques, 4,5-18 cm de long et $0,5-1,8 \mathrm{~cm}$ de large, à base décurrente, à sommet acuminé, à bord serré; face supérieure vert sombre, glabre; face inférieure blanchâtre, villeuse à tomenteuse, absence de poils glanduleux, à nervures brunes glabrescentes; pétiolules de 0-15 mm de long. Cymes terminales à subterminales, composées, trichotomes; pédoncule pouvant atteindre $3 \mathrm{~cm}$ de long, pubescent; pédicelles 2-6 $\mathrm{mm}$ de long, pubescents; bractées et bractéoles triangulaires, pouvant atteindre $10 \mathrm{~mm}$ de long, pubescents; bouton floral oblong, cylindrique, constricté à mi-hauteur, à élargissement supérieur légèrement plus épais que l'élargissement inférieur, à nombreux poils glanduleux; élargissement inférieur tomenteux. Fleurs d'environ $3 \mathrm{~mm}$ de long; calice cupuliforme, tomenteux, verdâtre, de 0,5-1 mm de long; corolle légèrement cylindrique, à 4 mamelons arrondis au sommet; pétale subtriangulaire à face externe rouge, à bord jaune verdâtre, à face interne à nombreux poils glanduleux; étamines 4 , d'environ $0,1 \mathrm{~cm}$ de long; disque constitué de 4 glandes coniques, roses. Baies noir violacé à maturité, ovoïdes, de $1,2 \mathrm{~cm}$ de long et $0,6 \mathrm{~cm}$ de large; graine de $0,8 \mathrm{~cm}$ de long et $0,5 \mathrm{~cm}$ de large, ellipsoïde, à crête ventrale irrégulière, peu saillante.

\section{ZAIRE :}

SHABA: Nzilo-Kyamasumba, km 11, vallée de la Luilu [E 2524', S $10^{\circ} 30^{\prime}$ ], alt. $1190 \mathrm{~m}$, forêt claire sur sol rocheux, janv. 1986, Bamps \&

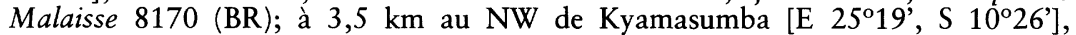
alt. $1025 \mathrm{~m}$, forêt claire, janv. 1988, Schaijes 3927 (BR); riv. Kambele [E $25^{\circ} 19^{\prime}$, S $10^{\circ} 45^{\prime}$ ], alt. $1425 \mathrm{~m}$, termitière en forêt claire, déc. 1974 , Malaisse 8418 (BR); Lubumbashi-Likasi, km 50, riv. Kamianga [E $27^{\circ} 17^{\prime}$, S $11^{\circ} 18^{\prime}$ ], alt. $1335 \mathrm{~m}$, flanc de ravin en forêt claire, janv. 1984, Malaisse 13090 (BR);

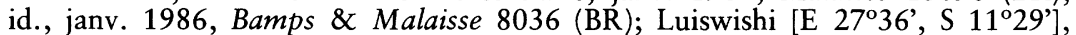
alt. $1208 \mathrm{~m}$, savane, janv. 1972, Malaisse 7134 (BR); id., janv. 1981, Malaisse 11417 (BR); id., janv. 1982, Malaisse 12661 (BR); id., fév. 1982, Malaisse \& Robbrecht 2020 (BR); id., mars 1983, Ndjele 1029 (BR); id., déc. 1984, Malaisse 13402 (BR).

Tanzanie, Zambie.

Fig. 1. - Cyphostemma septemfoliolatum Wild \& Drummond: A, port $(\times 1 / 6)$; $\mathrm{B}$, stipule $(\times 2)$; C, bouton floral $(\times 10) ; \mathrm{D}$, pétale $(\times 10)$; $\mathrm{E}$, fleur fanée, calice, corolle et étamines ôtés $(\times 14) ; \mathrm{F}$, fruit $(\times 3)$; G, graine, vue de profil $(\times 7) ; \mathrm{H}$, graine, vue ventrale $(\times 7)$; I, graine, vue dorsale $(\times 7)$ (A-B d'après Malaisse 12661, C-E d'après Malaisse 13402 bis, F-I d'après Ndjele 1029). 


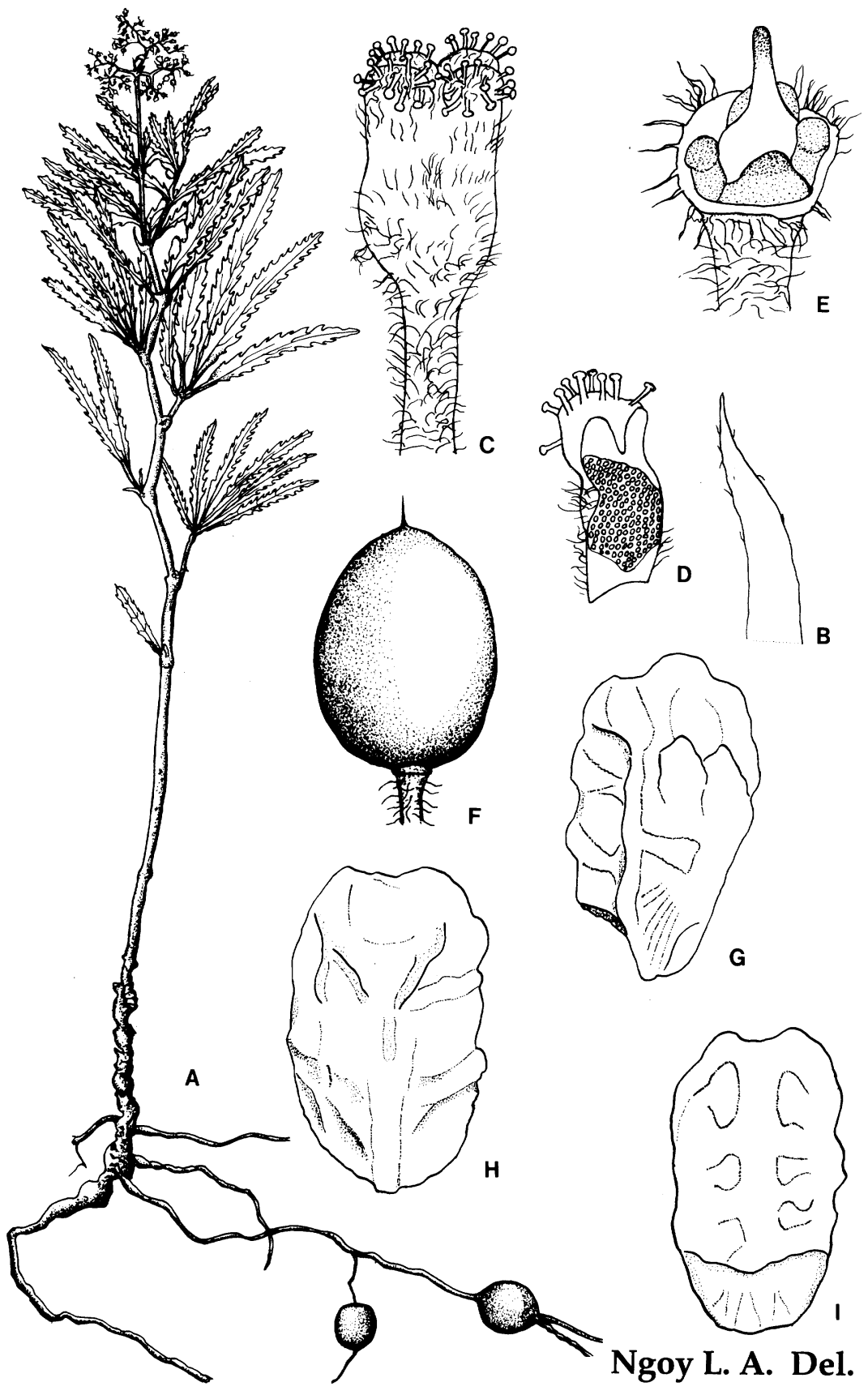


Habitat: Forêts claires de type miombo humide et savanes arborées à mauvaise économie en eau.

Observations: 1. - Plante nouvelle pour le Zaïre.

2. - Floraison en décembre, fructification en janvier-février.

3. - La présence de vrilles a été exceptionnellement notée à la base de l'inflorescence (Malaisse 8418) ou des feuilles (Bamps \& Malaisse 8710).

4. - Le matériel du Shaba présente une corolle glanduleuse et pubescente, tandis que le matériel de Zambie et de Tanzanie est glabre. Wild \& Drummond (Fl. Zamb. 2: 483. 1966) notaient à propos de Cyphostemma mildbreadii: "It is unusual for a Cyphostemma species in that it appears to show considerable variation in two characters that are normally fairly constant in the genus, i.e., the presence or absence of tendrils and the presence or absence of glands on the corolla». Ce commentaire s'applique aussi à C. septemfoliolatum. Notre étude des Cyphostemma en provenance du Shaba montre que cette variabilité est moins exceptionnelle pour ce genre que Wild \& Drummond (op. cit.) ne le supposaient.

2. Cyphostemma nanellum (Gilg \& Fr.) Descoings, Not. Syst. 16: 123 (1960); Wild \& Drummond, Fl. Zamb. 2: 470 (1966). - Fig. 2.

Cissus nanella Gilg \& Fr. in R.E. Fr., Schwed. Rhod.-KongoExped. 1: 135, tab. 10 [4] (1914).

Herbe vivace, dressée, pouvant atteindre $25 \mathrm{~cm}$ de haut, à racine principale pivotante pouvant atteindre $1,5(2) \mathrm{cm}$ de diam. et $3-9 \mathrm{~cm}$ de longueur, prolongée par une racine fine, racines latérales peu nombreuses; tige grêle, pubescente; vrilles absentes. Feuilles 2-4 à (2)3 folioles digitées; pétioles pouvant atteindre $3,5 \mathrm{~cm}$ de long, à même indument que la tige, portant en outre quelques glandes courtement pédicellées à proximité du sommet; stipules triangulaires à lancéolées, à sommet acuminé, à bord pubescent, pouvant atteindre $7 \mathrm{~mm}$ de long et $3 \mathrm{~mm}$ de large; folioles linéaires à étroitement oblancéolées, sessiles, à bord entier dans les $2 / 3$ inférieurs, irrégulièrement denté à serré pour le $1 / 3$ supérieur, de $5-7 \mathrm{~cm}$ de long et $0,8-2,5 \mathrm{~cm}$ de large, à face supérieure glabre, à face inférieure glabre à l'exception de la 

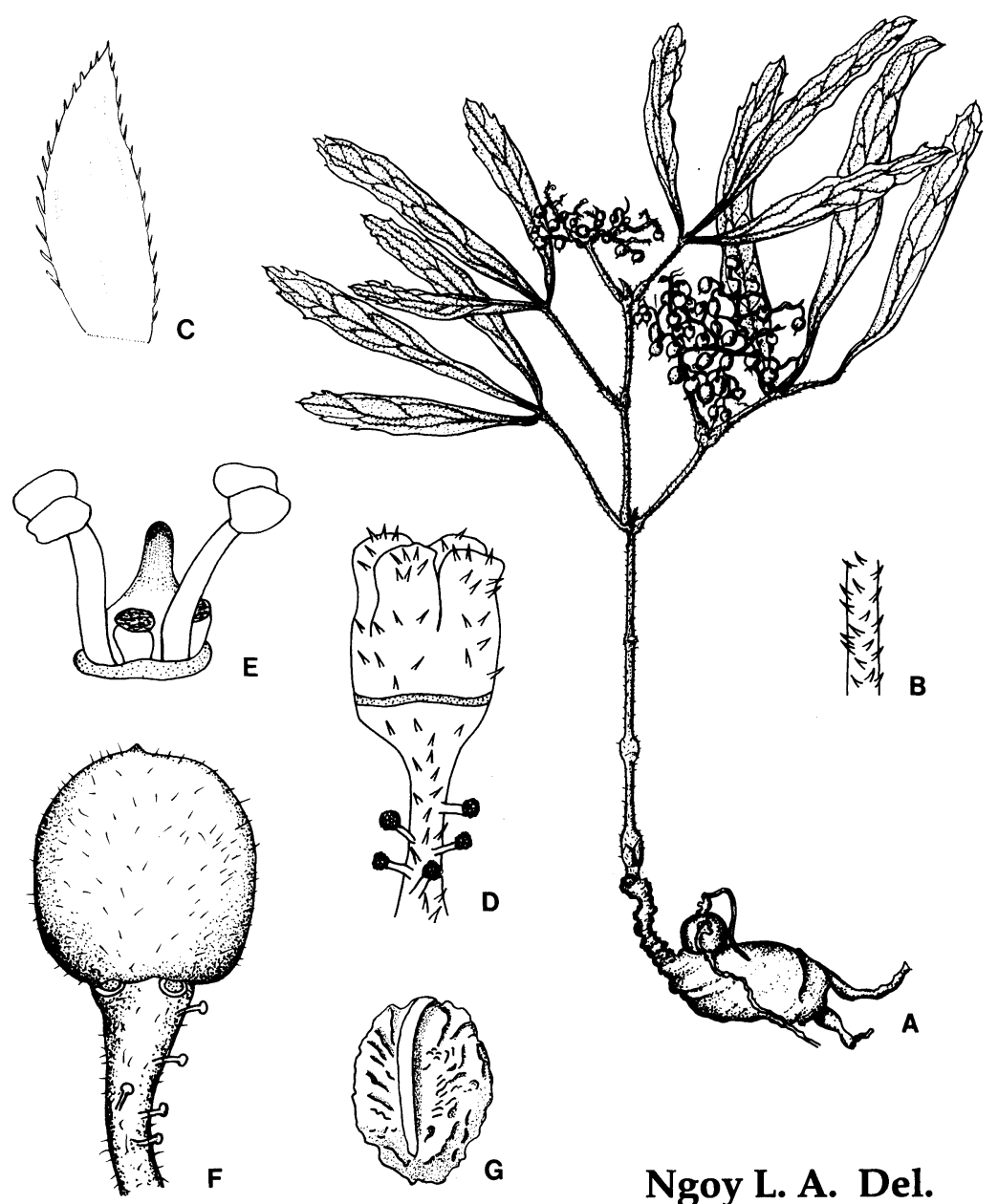

Ngoy L. A. Del.

Fig. 2. - Cyphostemma nanellum (Gilg \& Fries) Descoings: A, port $(\times 1 / 3)$; $\mathrm{B}$, indument de la tige $(\times 3)$; $\mathrm{C}$, stipule $(\times 5)$; $\mathrm{D}$, bouton floral $(\times 10)$; $\mathrm{E}$, fleur, calice et corolle ôtés ( $\times 12) ; \mathrm{F}$, fruit $(\times 4,5) ; G$, graine, vue ventrale ( $\times 5$ ) (A-E d'après Malaisse 12818, F-G d'après Malaisse 13419).

nervure principale pubescente. Cymes axillaires et (ou) terminales, composées, trichotomes; pédoncule $1-2 \mathrm{~cm}$ de long, pubescent; pédicelle 2-3 $\mathrm{mm}$ de long, pubescent et portant des poils glanduleux; bouton floral pubescent, pouvant atteindre $3,5 \mathrm{~mm}$ de long, oblong-cylindrique, légèrement rétréci à mi-hauteur. Fleurs à sépales à bord pourpre, pubérulent, de $0,5 \mathrm{~mm}$ de long; pétales de $3,5 \mathrm{~mm}$ de long; étamines de $3-3,5 \mathrm{~mm}$ de long; ovaire 
pubérulent, à style de $1 \mathrm{~mm}$ de haut. Baies ovoïdes, de 5,5$6 \mathrm{~mm}$ de long, de $5 \mathrm{~mm}$ de large, pubescente à glabrescente; graine de $4 \mathrm{~mm}$ de long et $3 \mathrm{~mm}$ de large, ellipsoïde, à crête ventrale rectiligne et saillante.

\section{ZAÏRE :}

SHABA : Kingandu [E $27^{\circ} 20^{\prime}$, S $11^{\circ} 48^{\prime}$ ], alt. $1250 \mathrm{~m}$, forêt claire, déc. 1985 , Malaisse \& Matamba 11 (BR); id., janv. 1985, Malaisse 13419 (BR); Luiswishi

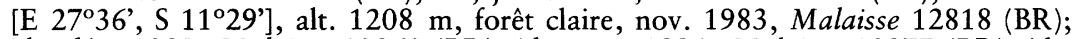
id., déc. 1983, Malaisse 12868 (BR); id., nov. 1984, Malaisse 13277 (BR); id., déc. 1984, Malaisse 13412 (BR); Kasapa [E $27^{\circ} 28^{\prime}, S 11^{\circ} 35^{\prime}$ ], alt. $1245 \mathrm{~m}$, forêt claire, fév. 1982, Malaisse \& Robbrecht 2079 (BR); id., nov. 1984, Matamba 37 (BR).

Zambie.

Habitat: Forêts claires à Marquesia macroura Gilg et Julbernardia globiflora (Benth.) Troupin ainsi que forêts claires de type miombo humide.

Observations: 1. - Plante nouvelle pour le Zaïre.

2. - Floraison en novembre, fructification en janvier.

3. Cyphostemma viscosum (Gilg \& Fr.) Descoings, Not. Syst. 16: 125 (1960); Wild \& Drummond, Fl. Zamb. 2: 470 (1966).

Cissus viscosa Gilg \& Fr. in R.E. Fr., Schwed. Rhod.-KongoExped. 1: 136 (1914).

Herbe dressée, d'environ $1 \mathrm{~m}$ de haut; racine principale ligneuse, puissante, racines secondaires fusiformes; tige robuste, à section circulaire, pourvue de glandes capitées sessiles et de glandes pédicellées; vrilles absentes. Feuilles trifoliolées, digitées; stipules pouvant atteindre $3 \mathrm{~cm}$ de long, ovales-acuminées, visqueuses, couvertes de glandes sessiles, caduques; pétiole $2-5 \mathrm{~cm}$ de long, 6-8 $\mathrm{mm}$ de diam., indument semblable à celui de la tige; pétiolule $1-3 \mathrm{~cm}$ de long; folioles pouvant atteindre $45 \mathrm{~cm}$ de long et $25 \mathrm{~cm}$ de large, étroitement obovales, obovales ou elliptiques, à sommet aigu à obtus, à bord crénelé ou ondulé-crénelé, à base cunée, discolores, à face supérieure vert sombre, glabre à l'exception de quelques glandes sessiles produisant un aspect vernissé, 
face inférieure vert grisâtre à blanchâtre. Cymes terminales, trichotomes; pédoncule pouvant atteindre $8 \mathrm{~cm}$ de long, à indument semblable à celui de la tige; pédicelles de $2 \mathrm{~mm}$ de long, pubérulent et glanduleux; bractées et bractéoles de 1-2 mm de long, subulées, pubéruleuses; bouton floral de $3 \mathrm{~mm}$ de long, oblongcylindrique, rétréci juste au-dessus de la mi-hauteur, pubérulent et à glandes subsessiles. Fleurs à calice de $0,5 \mathrm{~mm}$ de long, pubérulent et glanduleux; pétales verts; ovaire glanduleux-pubérulent; style de 1,5 mm de long, à sommet subcapité. Fruit violetnoirâtre, de $1,8 \mathrm{~cm}$ de long et $1,2 \mathrm{~cm}$ de diam. à l'état frais, ellipsoïde, décroissant légèrement vers le sommet, pubérulent et glanduleux, à glandes subsessiles et glandes pédicellées; graine 1, de $8 \mathrm{~mm}$ de long et $5 \mathrm{~mm}$ de diam., ellipsoïde, à crête dorsale rugueuse et paroi lisse.

ZAÏRE :

SHABA: $3 \mathrm{~km} \mathrm{~S}$ de Tenke [E 2607', S 10³8'], alt. $1380 \mathrm{~m}$, forêt claire sur sol sableux, déc. 1984, Malaisse 13403 ( $\mathrm{B}, \mathrm{BR}, \mathrm{C}, \mathrm{EA}, \mathrm{MO}, \mathrm{P}, \mathrm{PRE}$, WAG).

Zimbabwe, Zambie.

Habitat: Forêts claires.

Observations: 1. - Plante nouvelle pour le Zaïre.

2. - La présence de sphères tubéreuses, fréquentes chez les Cyphostemma du Shaba, n'a pas été recherchée.

3. - Nous n'avons pas vu du matériel fleuri en provenance du Zaïre.

4. Cyphostemma vandenbergheae Malaisse \& Matamba sp. nov.; C. alnifolio (Schweinf. ex. Planch.) Descoings affine, sed folii nervatione reticulato-pinnata versus brochidodroma, alabastrorum foliorumque trichomatibus valde differt. - Fig. 3.

Herba perennis erecta, $30-50 \mathrm{~cm}$ alta; radix cylindrica, radicibus secundariis inflatis, stipite semi-lignoso valido; caulis cylindricus, striatus, puberulus et pilis rubro-glandulosis densis obtectus, pilis glandulosis ad basim plantae densissimis usque ad $20 \mathrm{~mm}$ longis sed ad apicem plantae brevioribus; cirrhi deficien- 
tes. Folia 3-5, alterna, sessilia, simplicia, reticulato-pinnata, chartacea, pubescentia; stipulae triangulares, $20-28 \mathrm{~mm}$ longae et 4-5 $\mathrm{mm}$ latae, pagina superior glabra, pagina inferior dense puberula; lamina triangularis ad ovata, usque ad $25 \times 18 \mathrm{~cm}$, apice acuta, basi breve angustata vel truncata, irregulariter duplicatoserrata, pagina superior sparse puberula et pilis glandulosis brevibus sparse donata, pagina inferior dense puberula et pilis glandulosis longis sparse donata, sed ad margines pilis simplicibus et luteo-glandulosis ornata. Cymae compositae usque ad $15 \mathrm{~cm}$ longae, pedunculo usque ad $8 \mathrm{~cm}$ longo; pedunculus et axes pubescentes et pilis glandulosis donati; bractae et bracteolae triangulares, ca. 1,2 $\mathrm{mm}$ longae et $0,4 \mathrm{~mm}$ latae, ad margines pilis longis ornatae, pagina superior pilis longis ornata, pagina inferior glabra. Flores pedicello ca. $3 \mathrm{~mm}$ longo pubescenti et pilis glandulosis donato; alabastrum cylindricum, ad $1 / 3$ superiorem constrictum, apice paulum inflatum, ca. $2 \mathrm{~mm}$ longum et $1,7 \mathrm{~mm}$ latum; calyx ca. 0,5 mm longus, cupuliformis, subinteger, pubescens; petala pagina inferior glabra sed ad marginem linea pilorum et apice pilorum coma donata; stamina ca. $1 \mathrm{~mm}$ longa; antherae $0,3 \mathrm{~mm}$ longae; ovarium ca. $2 \mathrm{~mm}$ longum, pilis ornatum; stylus ca. $2 \mathrm{~mm}$ longus; stigma indistinctum. Baccae ignotae.

\section{ZAÏRE :}

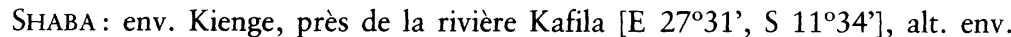
$900 \mathrm{~m}$, savane arborée, nov. 1988, M. Vandenberghe 131 (BR, alab.); id., déc. 1989, M. Vandenberghe 171 (BR, fl., fr. juven.), 172-173 (BR, alab.), 174 (BR, fl., holotype); Gombela - plateau des Kundelungu km 20 [E 27\%49', S 1040'], alt. env. $1400 \mathrm{~m}$, savane arborée, déc. $1989, \mathrm{M}$. Vandenberghe 179 (BR, alab.); id., M. Vandenberghe 180 (BR, f.).

\section{Habitat: Savanes arborées.}

Observations: Nous nous faisons un plaisir de dédier cette espèce à Mme Moniek Vandenberghe, qui récolta cette plante et

Fig. 3. - Cyphostemma vandenbergheae Malaisse \& Matamba: A, partie souterraine $(\times 2 / 5)$; B, port $(\times 2 / 5)$; C, poils de la tige $(\times 1) ; \mathrm{D}$, feuille $(\times 2 / 5)$; E, bord du limbe, face supérieure $(\times 4)$; F, stipule, face supérieure $(\times 8 / 5) ; \mathrm{G}$, id., face inférieure $(\times 8 / 5) ; \mathrm{H}$, bouton floral $(\times 9) ; \mathrm{I}$, pétale, vue latérale $(x 18)$; J, id., vue ventrale $(\times 18)$; K, id., vue dorsale $(\times 18)$ (A-B, D-G d'après $M$. Vandenberghe 179 , C d'après $M$. Vandenberghe $180, \mathrm{H}-\mathrm{K}$ d'après $M$. Vandenberghe 174). 


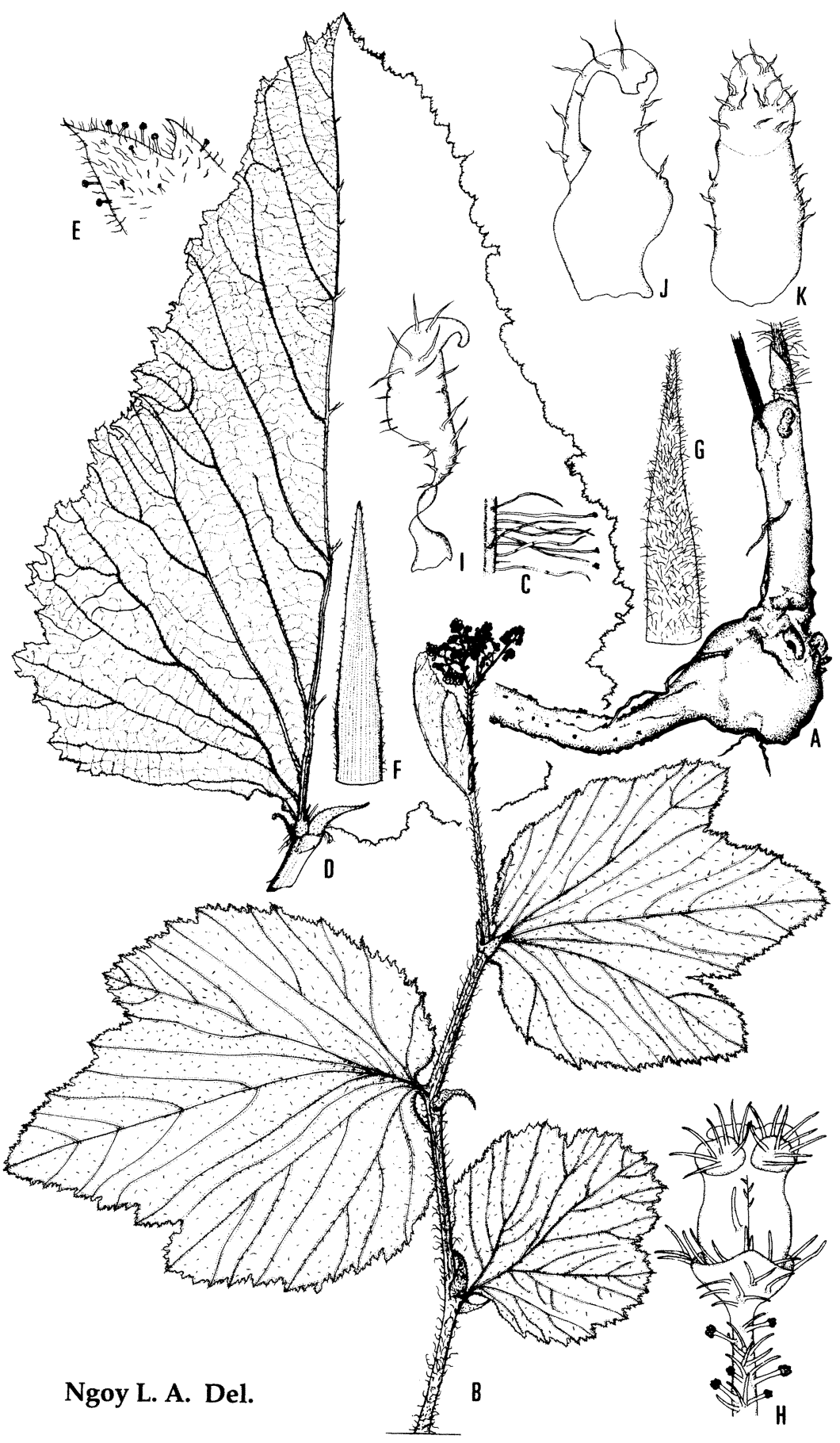


dont la collection de Cyphostemma du Shaba a largement contribué à la connaissance de ce genre dans la dition.

Cette nouvelle espèce est remarquable par la variabilité de la forme du limbe sur un même individu. En 1960, Dewit (Fl. Congo Belge Ruanda-Urundi 9: 470. 1960) ne signalait qu'une seule espèce à feuilles simples dans la section Cyphostemma Planch. du genre Cissus L. au Zaïre. Les Cyphostemma à feuilles simples du territoire de la Flore d'Afrique centrale peuvent être identifiés par la clef de détermination suivante:

Plantes rampantes:

Vrilles absentes; feuilles ovales à elliptiques, glabres à la face supérieure; absence de poils glanduleux. . . . . . . . . . . . . C. wittei Vrilles présentes; feuilles cordées; poils glanduleux sur la tige, les vrilles et les pédoncules. . . . . . . . . . . . C. kundelunguense

Plantes dressées:

Feuilles, tige, pédoncules et boutons floraux glabres . . C. simplicifolium Feuilles, tige, pédoncules et boutons floraux pubescents .

C. vandenbergheae

Remerciements: Les auteurs remercient vivement Kisimba $\mathrm{K}$. M. et Muzinga Y. K. pour leur aide efficace sur le terrain, Ngoy L. A. pour le dessin du port des trois espèces figurées et la mise au net des dessins de détail. Nous sommes encore reconnaissant à Messieurs T. Müller et R.B. Drummond (SRGH) ainsi qu’à Monsieur Petit (BR) pour nous avoir facilité l'accès aux riches collections de leur Institution. Madame le Professeur M. Bourgeois, de l'Université de Lubumbashi, a mis à notre disposition une loupe binoculaire, notre gratitude lui est acquise. 